\title{
Piezoelectric $\mathrm{BaTiO}_{3}$ Thin Film Nanogenerator on Plastic Substrates
}

\author{
Kwi-Il Park, ${ }^{\dagger}$ Sheng Xu, ${ }^{\mp}$ Ying Liu, ${ }^{\ddagger}$ Geon-Tae Hwang, ${ }^{\dagger}$ Suk-Joong L. Kang, ${ }^{\dagger}$ \\ Zhong Lin Wang, ${ }^{\ddagger}$ and Keon Jae Lee* ${ }^{\dagger}$
}

${ }^{\dagger}$ Department of Materials Science and Engineering, Korea Advanced Institute of Science and Technology (KAIST), 373-1 Gwahangno, Yuseong-gu, Daejeon 305-701 Republic of Korea, and ${ }^{*}$ School of Materials Science and Engineering, Georgia Institute of Technology, 771 Ferst Drive, Atlanta, Georgia 30332-0245, United States

\begin{abstract}
The piezoelectric generation of perovskite $\mathrm{BaTiO}_{3}$ thin films on a flexible substrate has been applied to convert mechanical energy to electrical energy for the first time. Ferroelectric $\mathrm{BaTiO}_{3}$ thin films were deposited by radio frequency magnetron sputtering on a Pt/Ti/ $/ \mathrm{SiO}_{2} /(100) \mathrm{Si}$ substrate and poled under an electric field of $100 \mathrm{kV} / \mathrm{cm}$. The metal-insulator $\left(\mathrm{BaTiO}_{3}\right)-\mathrm{metal}_{\text {-structured }}$ ribbons were successfully transferred onto a flexible substrate and connected by interdigitated electrodes. When periodically deformed by a bending stage, a flexible $\mathrm{BaTiO}_{3}$ nanogenerator can generate an output voltage of up to $1.0 \mathrm{~V}$. The fabricated nanogenerator produced an output current density of $0.19 \mu \mathrm{A} / \mathrm{cm}^{2}$ and a power density of $\sim 7 \mathrm{~mW} / \mathrm{cm}^{3}$. The results show that a nanogenerator can be used to power flexible displays by means of mechanical agitations for future touchable display technologies.
\end{abstract}

KEYWORDS $\mathrm{BaTiO}_{3}$, thin film, piezoelectric, flexible electronics, nanogenerator, energy harvesting

$\mathrm{E}$ nergy harvesting technologies that convert existing sources of energies, such as thermal energy as well as vibrational and mechanical energy from the natural sources of wind, waves, or animal movements into electrical energy, is attracting immense interest in the scientific community. ${ }^{1-6}$ The fabrication of nanogenerators is particularly interesting because it can even scavenge the biomechanical energy from inside the human body, such as the heart beat, blood flow, muscle stretching, or eye blinking, and turn it into electricity to power implantable biodevices. ${ }^{7-9}$

One way of harvesting electrical energy from the mechanical energy of ambient vibrations is to utilize the piezoelectric properties of ferroelectric materials. Piezoelectric harvesting has been proposed and investigated by many researchers. ${ }^{10-14}$ Chen et al. ${ }^{12}$ reported on the fabrication of a nanogenerator that involves the use of lead zirconate titanate $\left(\mathrm{PbZr}_{x} \mathrm{Ti}_{1-x} \mathrm{O}_{3}, \mathrm{PZT}\right)$ nanofibers on a bulk Si substrate. The PZT nanofibers were connected to interdigitated electrodes (IDEs) and, when pressure was applied perpendicularly to the nanogenerator surface, the nanogenerator produced an outstanding output voltage. Wang and co-workers ${ }^{13,14}$ used piezoelectric ZnO nanowires to develop a multiple lateral-nanowire-array integrated nanogenerator (LING) ${ }^{13}$ and a high-output nanogenerator (HONG) ${ }^{14}$ on plastic substrates. They also demonstrated the feasibility of harvesting energy from the breath and heartbeat of animals. ${ }^{9}$ As of today, the nanogenerator has an output voltage of $2 \mathrm{~V}$, and the power generated can be used to power a commercial

* To whom correspondence should be addressed. E-mail: keonlee@kaist.ac.kr. Phone: 82-42-350-3343. Fax: 82-42-350-3310.

Received for review: 08/20/2010

Published on Web: 00/00/0000 light-emitting diode (LED) ${ }^{14}$ Recently, there have been attempts to transfer flexible perovskite materials and capacitors onto flexible substrates for the purpose of utilizing the high inherent piezo-properties of ferroelectric materials from bulk substrates. ${ }^{15,16}$ In those attempts, perovskite thin films (PZT and $\mathrm{BaTiO}_{3}$ ) deposited on bulk substrates were annealed at high temperatures and transferred onto plastic substrates by the removal of the sacrificial layers ( $\mathrm{MgO}$ and $\mathrm{TiO}_{2}$ ).

This work reports on the first use of lead-free biocompatible $\mathrm{BaTiO}_{3}$ in the fabrication and characterization of a nanogenerator on a flexible substrate. A perovskite ceramic$\mathrm{BaTiO}_{3}$ thin film deposited on a $\mathrm{Pt} / \mathrm{Ti} / \mathrm{SiO}_{2} /(111) \mathrm{Si}$ substrate by rf magnetron sputtering was annealed at $700{ }^{\circ} \mathrm{C}$ for crystallization and poled to enhance the high piezoelectric property. The $\mathrm{BaTiO}_{3}$ thin film was then transferred onto a flexible substrate by means of standard microfabrication and soft lithographic printing techniques. ${ }^{17-20}$ To measure the positive and negative output voltage/current signals, we deposited the IDEs on flexible $\mathrm{BaTiO}_{3}$.

Figure 1 a shows a schematic of the fabrication steps. (i) Deposition of an amorphous $\mathrm{BaTiO}_{3}$ thin film on a Pt/Ti/SiO $2 /$ Si substrate. A $300 \mathrm{~nm} \mathrm{BaTiO}$ thin film was deposited on a $\mathrm{Pt} / \mathrm{Ti} / \mathrm{SiO}_{2} / \mathrm{Si}$ substrate by rf magnetron sputtering at room temperature in an $\mathrm{Ar}$ atmosphere. The $\mathrm{BaTiO}_{3}$ thin film was then annealed at $700{ }^{\circ} \mathrm{C}$ for $15 \mathrm{~min}$ in oxygen using the rapid thermal annealing (RTA) for the crystallization of the amorphous film. (ii) Inductive coupled plasma-reactive ion etcher (ICP-RIE) etching of metal-insulator-metal (MIM) structures $\left(\mathrm{Au} / \mathrm{BaTiO}_{3} / \mathrm{Pt}\right.$ layers). The MIM structure was etched by chlorine gas based ICP-RIE etching using an Al and plasma enhanced chemical vapor deposited-SiO $\mathrm{S}_{2}$ (PEO) mask made with a narrow bridge pattern $(300 \mu \mathrm{m} \times 50 \mu \mathrm{m})$. 

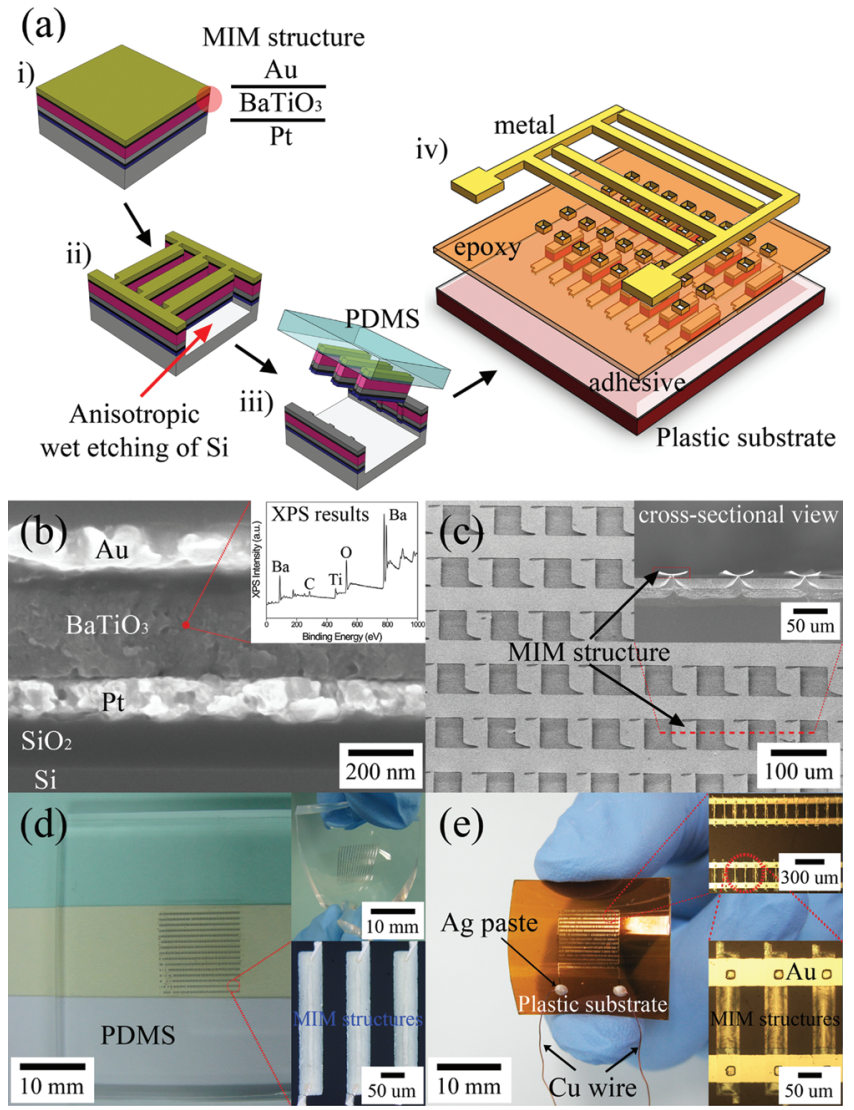

FIGURE 1. (a) Schematic illustration of the process for fabricating flexible $\mathrm{BaTiO}_{3}$ nanogenerator on plastic substrates. (b) A crosssectional SEM image of a MIM structure $\left(\mathrm{Au} / \mathrm{BaTiO}_{3} / \mathrm{Pt}\right.$ layers) on an $\mathrm{SiO}_{2} / \mathrm{Si}$ substrate. The inset shows an XPS spectrum obtained from an annealed $\mathrm{BaTiO}_{3}$ thin film. (c) SEM photograph of the MIM structure $\left(\mathrm{Au} / \mathrm{BaTiO}_{3} / \mathrm{Pt}\right)$ on an $\mathrm{SiO}_{2} / \mathrm{Si}$ substrate after anisotropic etching of the Si layer at $80^{\circ} \mathrm{C}$ with $5 \%$ TMAH (tetramethylammonium hydroxide) for $12 \mathrm{~min}$. The inset shows the cross-sectional SEM image (corresponding to Figure 1a-ii) of the patterned MIM structures that are partially supported by the Si substrates during etching. (d) Photograph and magnified optical image of a PDMS slab inked with MIM structures. The inset indicates that the MIM structures $(300 \mu \mathrm{m}$ $\times 50 \mu \mathrm{m}$ ) were successfully transferred onto the elastomer. (e) A flexible $\mathrm{BaTiO}_{3}$ nanogenerator (with an area of about $82 \mathrm{~mm}^{2}$ area and a fill factor of $16.4 \%$ ) supported on a plastic substrate after the PDMS is peeled off. The copper $(\mathrm{Cu})$ wires were fixed to metal pads by silver (Ag) paste for the purpose of measuring the output voltage. The inset shows a magnified optical image of the MIM structures connected to IDEs $(\mathrm{Au})$.

Anisotropic wet etching with $5 \%$ tetramethylammonium hydroxide (TMAH, $80^{\circ} \mathrm{C}$ for $18 \mathrm{~min}$ ) removed the underlying Si layer and separated the MIM structure ribbons from the mother substrates. (iii) Transfer of the MIM structures onto a plastic substrate. For the transfer, we placed a polydimethylsiloxane (PDMS) stamp (Sylgard 184, Dow Corning) in uniform contact with the top surface of the freestanding MIM structures. Upon quick removal from the Si wafer, the narrow bridge-shaped MIM structures were transferred onto the PDMS. An elastomer slab, inked with MIM structures, was then placed on a polyurethane (PU)-coated plastic substrate (Kapton film, $100 \mu \mathrm{m}$ in thickness). The PU was optically cured by a ultraviolet (UV) light. MIM structures were well settled on a plastic substrate when the PDMS was peeled away. (iv) Fabrication of self-powered flexible devices. The diluted UV sensitive epoxy (SU8) was spin-coated on top of $\mathrm{BaTiO}_{3} / \mathrm{PU} /$ plastic substrates and the metal contact area was then opened with a standard photolithography process. MIM structures in nanogenerator were connected with IDEs (Au) and poled at $150{ }^{\circ} \mathrm{C}$ by applying an electric field of $200 \mathrm{kV} / \mathrm{cm}$ for about $15 \mathrm{~h}$ (see Supporting Information for details on the fabrication of a flexible $\mathrm{BaTiO}_{3}$ nanogenerator on plastic substrates, Figure S1).

Figure $1 \mathrm{~b}$ shows a cross-sectional scanning electron microscopy (SEM) image of a MIM structure $\left(\mathrm{Au} / \mathrm{BaTiO}_{3} / \mathrm{Pt}\right.$ layers). The X-ray photoelectron spectroscopy (XPS, Al K $\alpha$ source) spectrum of the annealed $\mathrm{BaTiO}_{3}$ film in the inset consists mainly of $\mathrm{Ba}, \mathrm{Ti}$, and $\mathrm{O}$ peaks with a minor $\mathrm{C}$ peak; the latter might be due to the $\mathrm{C}$ contamination of the sample during its exposure in air. 21,22 Figure 1c shows an SEM image of MIM structures obtained at the intermediate stage of partial anisotropic etching of the underlying Si layer using TMAH etchant. The inset is a magnified cross-sectional view of the MIM structures. Note the mechanical flexibility of the $\sim 550 \mathrm{~nm}$ thick MIM structures. The photograph in Figure 1d (corresponding to Figure 1a-iii) shows MIM structures (of about $1 \mathrm{~cm}^{2}$ ) that were successfully transferred from a bulk Si wafer to a PDMS stamp without forming any cracks. The upper inset in Figure 1d shows a photograph of a twisted PDMS stamp that contains MIM structures; the bottom inset shows a magnified top view of the MIM structures on the PDMS stamp. Figure 1e (corresponding to Figure 1a-iv) displays a photograph and a magnified optical image of a flexible $\mathrm{BaTiO}_{3}$ nanogenerator device with a fill factor of about $16.4 \%$. The inset shows that the top and bottom electrodes of the MIM structures were connected by to the IDEs. Copper $(\mathrm{Cu})$ wires attached to the metal pads by a silver (Ag) paste were used to measure the output voltage and current.

We characterized the crystalline structure of the $\mathrm{BaTiO}_{3}$ thin films by X-ray diffraction (XRD) and Raman spectroscopy and measured the piezoelectric response by means of a piezoresponse force microscope (PFM). The XRD and Raman shift results indicate that the annealed $\mathrm{BaTiO}_{3}$ thin films on both bulk and flexible substrates have good crystallinity with a ferroelectric tetragonal phase (see Supporting Information for XRD and Raman analysis results of $\mathrm{BaTiO}_{3}$ thin films, Figure S2). As schematically shown in Figure 2a, the PFM technique was used to measure the piezoelectric constant, $d_{33}$ (the induced polarization per unit stress applied in an out-of-plane direction), of the $\mathrm{BaTiO}_{3}$ thin films on $\mathrm{Si}$ substrates. Figure $2 \mathrm{~b}$ presents the PFM results (in a plot of the piezoresponse amplitude versus the applied AC bias voltage) for poled and unpoled $\mathrm{BaTiO}_{3}$ thin films on $\mathrm{Si}$ substrates. As shown in the inset, the amplitudes of the piezoelectric response have a hysteresis loop over the applied voltage range. The piezoelectric coefficient $\left(d_{33}\right)$ is determined by the slope of the curve (piezoresponse/applied 
(a)

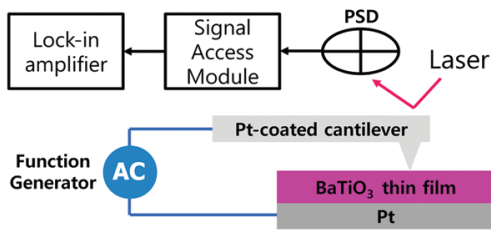

(b)

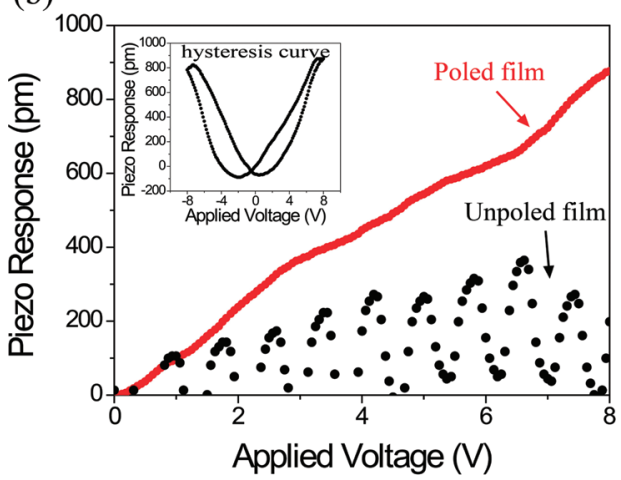

FIGURE 2. (a) Schematic of the PFM setup. (b) The piezoelectric response results of the $\mathrm{BaTiO}_{3}$ thin films before and after poling. The inset plots the piezoelectric property of a poled $\mathrm{BaTiO}_{3}$ thin film showing a hysteresis loop.

voltage). Without poling, the effective piezoelectric coefficient, $d_{33}$, of the $\mathrm{BaTiO}_{3}$ thin film on a $\mathrm{Pt} / \mathrm{Ti} / \mathrm{SiO}_{2} / \mathrm{Si}$ substrate was $40 \mathrm{pm} / \mathrm{V}$. After the poling at $140{ }^{\circ} \mathrm{C}$ under an electric field of $100 \mathrm{kV} / \mathrm{cm}$ for about $15 \mathrm{~h}, d_{33}$ increased to $105 \mathrm{pm} /$ $\mathrm{V}$. The measured result of the poled $\mathrm{BaTiO}_{3}$ thin film compares favorably with previously reported values for $\mathrm{BaTiO}_{3}$ thin film on Si substrates (that is, $d_{33}=30-100$ $\mathrm{pm} / \mathrm{V})^{23,24}$ and verifies that our process was well optimized.

Figure 3a-i,ii shows schematics of the appearance and cross-sectional structure of the poled $\mathrm{BaTiO}_{3}$ nanogenerator in its original state without bending. The piezoelectric material has dipoles that are aligned by poling under a high electric field. As depicted in the cross-sectional structure of the poled nanogenerator device in the original state of Figure $3 a$-ii, the dipoles in the $\mathrm{BaTiO}_{3}$ thin film were arrayed in a longitudinal direction with the surface of the device. When the nanogenerator was bent (corresponding to Figure 3aiii), charges were generated in each MIM structure due to the tensile stress induced by the deflection of the device (corresponding to Figure 3a-iv). The generated charges could then flow through the Au electrodes and build up an output voltage $(\Delta V)$ between the IDEs. When the nanogenerator was unbent, the electrons moved back in the opposite direction. ${ }^{2}$ Figure $3 b, c$ shows the measured output voltage and current of a flexible $\mathrm{BaTiO}_{3}$ nanogenerator containing $\sim 1350$ MIM structures during continual bending and unbending. To confirm that the output signal was generated by the bending of the MIM structures, a widely used switching-polarity test was also conducted. ${ }^{2,5,25}$ As shown in Figure $3 \mathrm{~b}$, when a current meter was forward connected to the nanogenerator, a positive voltage and current pulse was generated during fast bending of the substrates. In the case (a)
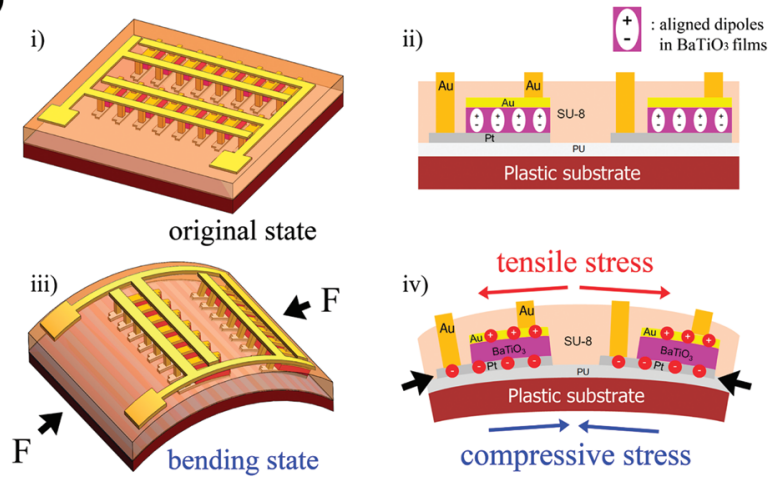

(b)
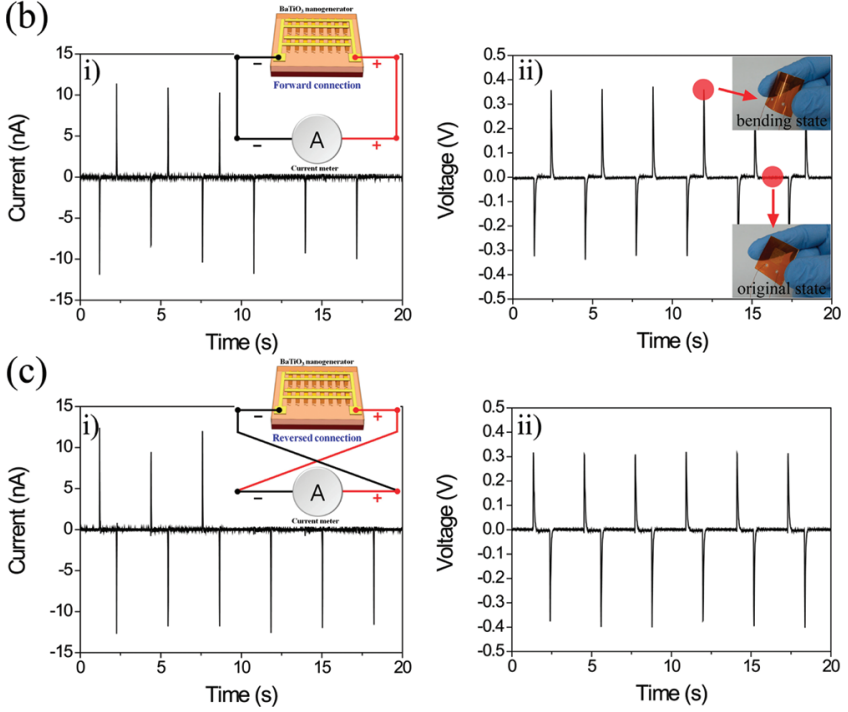

FIGURE 3. (a) Schematics of the power generation of the flexible $\mathrm{BaTiO}_{3}$ nanogenerator before and after bending. The dipoles are arrayed in a longitudinal direction of the device surface in the original state without bending (corresponding to Figure $3 a-i, i i)$. The charges are generated due to the tensile and compressive stresses induced by bending (corresponding to Figure 3a-iii,iv). The charges can move through the Au electrodes and build up an output voltage $(\Delta V)$ between the IDEs. The measured output current (left, i) and voltage (right, ii) of the $\mathrm{BaTiO}_{3}$ nanogenerator on a plastic substrate during periodic bending and unbending (b) when forward-connected (corresponding to the inset of Figure $3 \mathrm{~b}-\mathrm{i}$ ) to the current meter and (c) when reverse-connected (corresponding to the inset of Figure 3ci) to the current meter. The insets show optical images of nanogenerator in bent and unbent positions.

of the reverse connection (corresponding to Figure 3c), negative output signals were recorded. Under a repeated periodic motion of bending and unbending, the flexible $\mathrm{BaTiO}_{3}$ nanogenerator, which has a total area of about 82 $\mathrm{mm}^{2}$ and a fill factor of about $16.4 \%$, repeatedly generated an output voltage of $0.3-0.4 \mathrm{~V}$ and a current pulse of $8-12$ $\mathrm{nA}$; these values were created for a maximum strain of $0.40-0.55 \%$ at a straining rate of $1.2-1.6 \% \cdot \mathrm{s}^{-1}$. The amplitudes of the output voltage and current depend on the angular bending rate. During periodical bending/unbending of the nanogenerator with a finger, the maximum output voltage and current signal were $\sim 1.0 \mathrm{~V}$ and $\sim 26 \mathrm{nA}$, respectively (see Supporting Information for real time live 


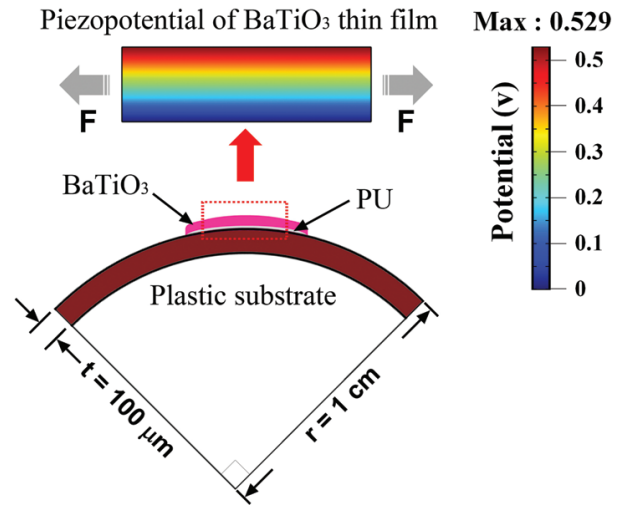

FIGURE 4. The calculated piezopotential distribution inside the $\mathrm{BaTiO}_{3}$ thin film. A pure tensile strain is assumed to exist in the thin film when the substrate is bent over $90^{\circ}$ with a radius of $1.0 \mathrm{~cm}$. The piezopotential difference inside the $\mathrm{BaTiO}_{3}$ thin film is 0.529 $\mathrm{v}$.

views and the output signals of bending experiments on energy harvesting, Video S1 and Figure S3), which are higher than the average values of nanogenerator using similar parallel electrodes. ${ }^{12,13}$ Furthermore, the output signals are expected to be improved by further optimizing, for example, fabrication process, material selection, ${ }^{15,26}$ the modified circuits, ${ }^{13,14}$ and three-dimensional (3D) integration structure. $^{27}$

Figure 4 shows the calculated piezopotential distributions inside the $\mathrm{BaTiO}_{3}$ thin film using a COMSOL software. We calculated the piezopotential distributions for a $\mathrm{BaTiO}_{3}(300$ $\mathrm{nm}) / \mathrm{PU}(2 \mu \mathrm{m}) /$ plastic substrate $(t=100 \mu \mathrm{m})$ with a bending radius of $R(1 \mathrm{~cm})$. A young's modulus of $E=67 \mathrm{GPa}^{28}$ and a piezoelectric coefficient of $d_{31}=78 \mathrm{pC} / \mathrm{N}^{28,29}$ were used for the finite element analysis (FEA). The strain $\left(\varepsilon_{t}\right)$ of the thin film is approximately equal to the strain $\left(\varepsilon_{\mathrm{s}}\right)$ of the outer surface of the plastic substrates due to thickness of the substrate is much larger than the thickness of the $\mathrm{BaTiO}_{3}$ thin film. ${ }^{2}$ According to the eq 1 , the calculated tensile strain of $\mathrm{BaTiO}_{3}$ thin film is $\sim 0.5 \%$.

$$
\varepsilon_{\mathrm{t}}=\varepsilon_{\mathrm{s}}=\frac{\Delta L}{L_{0}}=\frac{t}{2 R}
$$

The calculated results demonstrate that the tensile stress is $0.34 \mathrm{GPa}$ in the parallel direction. Although the lateral dimension (i.e., the length) of $\mathrm{BaTiO}_{3}$ thin films on plastic substrate influences the mechanics (tensile stress and surface strain) and the failure mode (cracking, slipping, or delamination) of films, ${ }^{30}$ for simplicity, we have ignored these effects, which might be insignificant, in our FEA simulation. The color code of Figure 4 represents the output piezopotential. The inside of the $\mathrm{BaTiO}_{3}$ thin film has a piezopotential difference of $0.53 \mathrm{~V}$, which agrees well to our measured output voltage.

In summary, we fabricated a $\mathrm{BaTiO}_{3}$ film based flexible nanogenerator. Annealed and poled ferroelectric $\mathrm{BaTiO}_{3}$ thin films were successfully transferred to a plastic substrate by means of conventional microfabrication and soft lithographic techniques. The $\mathrm{BaTiO}_{3}$ nanogenerator fabricated on a plastic substrate converted mechanical energy (bending) into electrical energy with an output voltage of up to $\sim 1.0 \mathrm{~V}$ and a current signal of up to $\sim 26 \mathrm{nA}$. The output current density was calculated to be $\sim 0.19 \mu \mathrm{A} / \mathrm{cm}^{2}$ and power density was calculated to be $\sim 7 \mathrm{~mW} / \mathrm{cm}^{3}$. The calculations are based on the total area and volume of the MIM structures. The output voltage agrees well to the theoretically calculated piezopotential. Our nanogenerator technique of transferring ferroelectric thin films from a bulk substrate to a flexible substrate demonstrates the feasibility of fabricating thin film nanogenerators with other perovskite-type oxide materials (e.g., $\mathrm{BiFeO}_{3}{ }^{26}$ and $\mathrm{PZT}^{15}$ ), which can exhibit higher piezoelectric performance than $\mathrm{BaTiO}_{3}$ and expanding the progressive application for energy harvesting devices. They also provide progressive opportunities for implantable biological devices (e.g., self-powered wireless biosensors and robots) due to the biocompatible material properties of $\mathrm{BaTiO}_{3}$ and have clear potential for use in electrical applications (e.g., artificial skin, LED, ${ }^{14}$ touchable piezoelectric displays, and piezotronics ${ }^{31}$ ), which are driven by pressure induced external sources.

Acknowledgment. This work was supported by the Basic Science Research Program (Grant 2010-0015466, CAFDC2010-0009903) and Fundamental R\&D Program for Core Technology of Materials (Grant 2010-0000739) through the National Research Foundation of Korea (NRF) funded by the Ministry of Education, Science, and Technology. Z.L.W. thanks the support from NSF, DARPA, and DOE.

Supporting Information Available. The detailed fabrication processes (Figure $\mathrm{S} 1$ ) of flexible $\mathrm{BaTiO}_{3}$ nanogenerator, the XRD and Raman shift results of $\mathrm{BaTiO}_{3}$ thin films (Figure S2), and the real time live views (Video S1) and the output signals (Figure S3) of experiment on power generation during periodical bending and unbending with a finger. This material is available free of charge via the Internet at http:// pubs.acs.org.

\section{REFERENCES AND NOTES}

(1) Tian, B. Z.; Zheng, X. L.; Kempa, T. J.; Fang, Y.; Yu, N. F.; Yu, G. H.; Huang, J. L.; Lieber, C. M. Nature 2007, 449 (7164), 885U8.

(2) Yang, R. S.; Qin, Y.; Dai, L. M.; Wang, Z. L. Nat. Nanotechnol. 2009, $4(1), 34-39$.

(3) Song, J. H.; Zhou, J.; Wang, Z. L. Nano Lett. 2006, 6 (8), 16561662 .

(4) Pan, C. F.; Wu, H.; Wang, C.; Wang, B.; Zhang, L.; Cheng, Z. D.; Hu, P.; Pan, W.; Zhou, Z. Y.; Yang, X.; Zhu, J. Adv. Mater. 2008, 20 (9), 1644-1648.

(5) Choi, D.; Choi, M. Y.; Choi, W. M.; Shin, H. J.; Park, H. K.; Seo, J. S.; Park, J.; Yoon, S. M.; Chae, S. J.; Lee, Y. H.; Kim, S. W.; Choi, J. Y.; Lee, S. Y.; Kim, J. M. Adv. Mater. 2010, 22 (19), 1-6.

(6) Goldschmidtboeing, F.; Woias, P. J. Micromech and Microeng. 2008, 18 (10), 104013.

(7) Wang, Z. L.; Song, J. H. Science 2006, 312 (5771), 242-246.

(8) Yang, R.; Qin, Y.; Li, C.; Zhu, G.; Wang, Z. L. Nano Lett. 2009, 9 (3), 1201-1205. 
(9) Li, Z.; Zhu, G. A.; Yang, R. S.; Wang, A. C.; Wang, Z. L. Adv. Mater 2010, 22 (23), 2534-2537.

(10) Wang, X. D.; Song, J. H.; Liu, J.; Wang, Z. L. Science 2007, 316 (5821), 102-105.

(11) Choi, M. Y.; Choi, D.; Jin, M. J.; Kim, I.; Kim, S. H.; Choi, J. Y.; Lee, S. Y.; Kim, J. M.; Kim, S. W. Adv. Mater. 2009, 21 (21), $2185-$ 2189

(12) Chen, X.; Xu, S. Y.; Yao, N.; Shi, Y. Nano Lett. 2010, 10 (6), 2133-2137.

(13) Xu, S.; Qin, Y.; Xu, C.; Wei, Y. G.; Yang, R. S.; Wang, Z. L. Nat. Nanotechnol. 2010, 5 (5), 366-373.

(14) Zhu, G.; Yang, R.; Wang, S.; Wang, Z. L. Nano Lett. 2010, 10 (8), $3151-3155$

(15) Qi, Y.; Jafferis, N. T.; Lyons, K.; Lee, C. M.; Ahmad, H.; McAlpine, M. C. Nano Lett. 2010, 10 (2), 524-528.

(16) Park, K. I.; Lee, S. Y.; Kim, S.; Chang, J.; Kang, S. J. L.; Lee, K. J Electrochem. Solid State Lett. 2010, 13 (7), G57-G59.

(17) Park, S. I.; Xiong, Y. J.; Kim, R. H.; Elvikis, P.; Meitl, M.; Kim, D. H.; Wu, J.; Yoon, J.; Yu, C. J.; Liu, Z. J.; Huang, Y. G.; Hwang, K.; Ferreira, P.; Li, X. L.; Choquette, K.; Rogers, J. A. Science 2009. 325 (5943), 977-981.

(18) Viventi, J.; Kim, D. H.; Moss, J. D.; Kim, Y. S.; Blanco, J. A.; Annetta, N.; Hicks, A.; Xiao, J. L.; Huang, Y. G.; Callans, D. J.; Rogers, J. A.; Litt, B. Sci. Transl. Med. 2010, 2 (24), 1-9.

(19) Lee, K. J.; Motala, M. J.; Meitl, M. A.; Childs, W. R.; Menard, E.; Shim, A. K.; Rogers, J. A.; Nuzzo, R. G. Adv. Mater. 2005, 17 (19), $2332-2336$

(20) Lee, K. J.; Lee, J.; Hwang, H. D.; Reitmeier, Z. J.; Davis, R. F.; Rogers, J. A.; Nuzzo, R. G. Small 2005, 1 (12), 1164-1168.
(21) Palchik, O.; Zhu, J. J.; Gedanken, A. J. Mater. Chem. 2000, 10 (5), $1251-1254$.

(22) Arai, M.; Kohiki, S.; Yoshikawa, H.; Fukushima, S.; Waseda, Y.; Oku, M. Phys. Rev. B 2002, 65 (8), No. 085101

(23) Park, Y. B.; Ruglovsky, J. L.; Atwater, H. A. Appl. Phys. Lett. 2004, 85 (3), 455-457.

(24) Guo, Y. P.; Suzuki, K.; Nishizawa, K.; Miki, T.; Kato, K. J. Cryst. Growth 2005, 284 (1-2), 190-196.

(25) Wang, X. D.; Liu, J.; Song, J. H.; Wang, Z. L. Nano Lett. 2007, 7 (8), 2475-2479.

(26) Zeches, R. J.; Rossell, M. D.; Zhang, J. X.; Hatt, A. J.; He, Q.; Yang, C. H.; Kumar, A.; Wang, C. H.; Melville, A.; Adamo, C.; Sheng, G.; Chu, Y. H.; Ihlefeld, J. F.; Erni, R.; Ederer, C.; Gopalan, V.; Chen, L. O.; Schlom, D. G.; Spaldin, N. A.; Martin, L. W.; Ramesh, R. Science 2009, 326 (5955), 977-980

(27) Ahn, J. H.; Kim, H. S.; Lee, K. J.; Jeon, S.; Kang, S. J.; Sun, Y. G.; Nuzzo, R. G.; Rogers, J. A. Science 2006, 314 (5806), 1754-1757.

(28) Sakakibara, T.; Izu, H.; Kura, T.; Shinohara, W.; lwata, H.; Kiyama, S.; Tsuda, S. Proceedings of IEEE 5 th International Symposium on Micro Machine and Human Science, October 2-4, 1994, Nagoya, Japan, p 75

(29) Jaffe, B.; Cook, W. R.; Jaffe, H. Piezoelectric Ceramics; Academic Press: London, 1971.

(30) Park, S. I.; Ahn, J. H.; Feng, X.; Wang, S. D.; Huang, Y. G.; Rogers, J. A. Adv. Funct. Mater. 2008, 18 (18), 2673-2684.

(31) Zhou, J.; Gu, Y. D.; Fei, P.; Mai, W. J.; Gao, Y. F.; Yang, R. S.; Bao, G.; Wang, Z. L. Nano Lett. 2008, 8 (9), 3035-3040 